\title{
Figurando una historia Della «teatralità» o «teatrabilità» del Decameron
}

\author{
Piermario Vescovo \\ Università Ca’ Foscari di Venezia \\ vescovo@unive.it
}

\begin{abstract}
A partire dal ruolo del Decameron quale repertorio novellistico di straordinaria fortuna — prima di tutto come serbatoio di trame - nella fondazione della commedia cinquecentesca, si affronta uno dei nodi essenziali dell'invenzione dello spazio comico del teatro all'italiana, dove la vita domestica e le azioni quotidiane sono raccontate e non agite sulle scena dai personaggi, per una convenzione fondativa che esclude la rappresentazione degli interni. Il confronto con la tradizione della visualizzazione della stessa dimensione nei disegni e nelle miniature dei manoscritti e nelle immagini dei libri a stampa si prova a diradare alcune leggende storiografiche su un inesistito "teatro medievale» impropriamente e fantasiosamente dedotto o inventato "guardando le figure». Questo permette di riflettere brevemente anche su alcune questioni di metodo, relativamente alla storia delle diverse discipline che incrociano i loro saperi e competenze a questo proposito.
\end{abstract}

Parole chiave: Boccaccio, Decameron, commedia rinascimentale, rappresentazione d'interni, miniature, immagini nei libri stampati.

\section{Abstract}

Since the Decameron, with its extraordinarily fortunate short-story style (first and foremost a source of plots) which lies at the foundation of $16^{\text {th }}$ century comedy, one of the essential hubs of invention has been faced within the space of comedy in Italian theater, where domestic life and day to day activities are told but do not act at the level of the characters, due to a fundamental convention that excludes the representations of the indoors. The confrontation between the tradition of showing that same dimension in the drawings, the manuscript miniatures and the images in the printed books proves the reduction in the frequency of some historiographic legends regarding the "medieval theater", which in fact never existed, and inappropriately and imaginatively developed or invented "watching the figures». This permits us to briefly reflect on some questions of method as well, with regard to the history of the various interwoven disciplines and their knowledge and competence in this matter.

Key words: Bocace, Decameron, $16^{\text {th }}$ century comedy, representations of the indoors, manuscript miniatures, images in the printed books. 
per Giorgio Padoan, dieci anni dopo

Tornando a vecchi interessi e a vecchie promesse non mantenute, ho realizzato quanto il tempo velocemente passi in rapporto al dialogo lasciato aperto con chi non c'è più. Queste persone sono Vittore Branca (che ha cercato più volte di invogliarmi alla fortuna teatrale boccacciana, fuori dal raggio ristretto del singolo rapporto testuale o intertestuale, cosa che ho, come capita nella vita, rinviando, sostanzialmente disatteso) e Giorgio Padoan.

La questione che vorrei qui provare ad affrontare è quella che (soprattutto nella bibliografia italiana o degli «italianisti») si suole indicare come della "teatralità del Decameron». Non ho tempo per spendermi ora in una critica alla gassosità della categoria di «teatralità» e lo farò solo indirettamente, partendo da qualche dettaglio concreto, dentro la storia della questione più ristretta che ora ci interessa.

Nel capitolo dedicato alla linea toscana della commedia cinquecentesca italiana, nel suo privilegiato rapporto col Decameron, nel suo ultimo e sistematico lavoro sull'argomento, Padoan pubblicava sotto all'illustrazione scelta per quelle pagine — una miniatura, relativa alla novella quarta della prima giornata, di un celebre manoscritto del Decameron (non indicato, ma si tratta di un prezioso manufatto, Fiandre, secondo quarto del XV secolo, conservato alla Bibliothèque dell'Arsenal) — la seguente didascalia: «Teatrabilità del Decameron». Lo stesso termine ritorna alla fine del volume, nell' Indice delle opere rinascimentali teatrali o teatrabili citate. ${ }^{1}$

Forse solo oggi, a una certa distanza (e a un decennio dalla scomparsa di Padoan), mi è chiaro quale impronta "anni settanta» —quelli in cui l'idea della «teatralità» era molto presente nella società e, di riflesso, anche negli studi- avesse la questione della "teatralità del Decameron». Ma soprattutto ho ritrovato una sostanziale solidarietà nelle prime righe in cui un Padoan giovane - trentunenne e non ancora studioso di testi teatrali- aveva scritto nel suo primo saggio di generale ricognizione boccacciana (quando era la questione dell' «ideologia» degli autori a tenere il campo) - Mondo aristocratico e mondo comunale nellideologia e nell'arte di Giovanni Boccaccio, 1964-, a proposito di un «intimo gusto della teatralità, in un'epoca in cui la produzione teatrale era ancora impensabile». ${ }^{2}$

Questo mi sembra - e il mio vuole essere un ricordo e un omaggio- il tratto che ha distinto la sua posizione, anche in quanto non esplicitamente espresso, rispetto ad altri richiami alla "teatralità» decameroniana, mostrati da saggi di indubbio rilievo come, in particolare, due capitoli di Realtà e stile nel "Decameron» di Mario Baratto (1970) e il contributo di Nino Borsellino intitolato Decameron come teatro (nel volume Rozzi e intronati: esperienze e forme di teatralità dal Decameron al Candelaio, 1974). La «teatralità» del Decameron

2. Così nell'originale redazione in rivista: cfr. Studi sul Boccaccio, II, 1964, p. 134. 
in questa, pur evidentemente legittima, prospettiva risiede, di fatto, in una proiezione di «scenicità», in una lettura cioè del centonovelle fatta con "gli occhi del teatro». Bisogna ovviamente vedere di quale teatro si trattasse. Si considerino i titoli dei due capitoli del libro di Baratto cui ho fatto ora allusione: Verso la commedia: il mimo e, senz'altro, La commedia. Ecco, in essi, il rilievo dato alla «vitalità del gesto e della parola in una precisa dimensione di spazio e tempo» e l'indicazione per cui «il lettore del Decameron diventa spettatore» (p. 240-241). Fissata questa relazione, il capitolo seguente si incentra sulla dichiarazione: «la novella del Boccaccio si costruisce nettamente per quadri scenici successivi: quasi una serie di atti dove predomina il dialogo, collegati con nessi di ordine narrativo» (p. 272).

Una domanda preliminare potrebbe riguardare il fatto se il principio di teatralità da queste pagine, peraltro assai raffinate, retrospettivamente proiettato sull'opera sia lo stesso principio che guidò (allorché il teatro ricominciò ad essere in Occidente una esperienza non più «impensabile») l'effettiva «teatralizzazione» del centonovelle. Questa domanda ne presuppone in realtà un'altra, e decisiva. Esiste una teatralità in atto o in potenza nel Decameron e se sì, quali sono i termini in cui un discorso storico (secondo un'istanza non totalmente coincidente con l'orizzonte di quella che per comodità possiamo chiamare la "critica letteraria») può provare a descriverla?

Non credo all' «immanenza dello spettacolo in seno al testo", già discutibile per il testo drammatico di potenziale o storicamente accertata destinazione scenica, e che rischia di essere fuorviante per il testo narrativo o poetico. Raccomanderei al proposito due lucidissime pagine di Ferdinando Taviani (persona che peraltro ha lungamente disputato a favore della «teatrabilità» contro la "teatralità») dove si individua con nettezza il principio minimo di insussistenza dell'elemento base di questo genere di analisi, nella figura o struttura tanto invocata ed evocata dei cosiddetti deittici ("questo»e «quello» e così via). Lascio la parola a Taviani:

Quando dall'oralità quotidiana i deittici passano in letteratura essi si pongono in una relazione cibernetica con il proprio contesto spazio-temporale. Ma questo spazio di reciproche implicazioni non è specialmente la scena teatrale. E la «scena» che non si può fare a meno di evocare ogni qualvolta si rievochi, si riproduca o si crei un dialogo, nel teatro e fuori del teatro. Nessuno, mi pare, ha dimostrato che i deittici compaiano nei dialoghi drammatici con maggior frequenza di quanto compaiano in dialoghi di romanzi o persino in dialoghi filosofici. $^{3}$

Che anche, più in là, il dialogo sia un principio di teatralità in atto risulta, per le medesime ragioni, altrettanto discutibile. Dove sta allora il principio che guida — se mai sommariamente e idealmente potessimo identificarlo- la futura fortuna teatrale del Decameron?

3. Ferdinando TaViani, "Attilia o lo spirito del testo", in Il magistero di Giovanni Getto. Lo statuto degli studi sul teatro, Genova: costa \& nolan, 1993, p. 217-286: p. 254. 
Boccaccio può contribuire solidamente all'invenzione di un teatro a venire perché alcuni elementi di una eredità teatrale a lunga gittata si raddensano in un'opera capitale, cent'anni prima dell'uscita dai lunghi secoli in cui la tradizione occidentale ha smarrito la nozione di modo teatrale. Egli sicuramente ignora il senso letterale della parola teatro, la sua esperienza concreta: basti aprire il Teseida dove, come in altri testi dell'età di mezzo, l'edificio che gli antichi chiamavano teatro risulta un luogo incomprensibile rispetto alla funzione di mettervi un tempo in scena delle storie, e serve, al più, come luogo d'incontro o in cui assistere a un torneo. Figurando una historia, racconta all'Averroè immaginato da Borges un mercante, relativamente a un rito incomprensibile che ha visto fare a degli uomini in una città lontana; all'Averroè che non riesce a connettere le parole della Poetica aristotelica al senso del gioco dei bambini sotto alla sua finestra.

Una più profonda necessità di allargare il quadro delle forme storicamente accertate di figurazione di una istoria muove questo piccolo intervento: spero sia evidente come l'altro enorme corno della questione sia costituito - e in rapporto al teatro ma anche e soprattutto per cercare di evitare la fantasia teatrale retrospettiva a partire da esso- dalla tradizione, proprio per la sua mole immensa, della visualizzazione del Decameron sulla pagina; tradizione che precede quella della sua teatralizzazione, ma in base a modalità diverse, se non opposte, da quelle che può sembrare legittimo immaginare col senso o l'esperienza culturale di poi.

Ma proviamo ad andare con ordine.

Un discorso sulla «teatralità» del Decameron, in atto o in potenza, può e deve farsi, dunque, solo tenendo presente la perdita delle nozioni di teatro e modo teatrale a cui lo stesso Boccaccio partecipa, pena l'inessenzialità (almeno l'inessenzialità storica) dell'operazione. Si tratta di un'istanza banale ma irrinunciabile, che porrò come prima opzione da condividere con chi mi vorrà seguire oltre in questo mio ragionamento.

La possibilità di imitare «letterariamente» le commedie antiche, soprattutto quelle terenziane, è una solida tradizione che attraversa la storia dei cosiddetti «secoli senza teatro». Gli autori di queste imitazioni possono addirittura nello spazio della pagina (o della mente) rivolgersi stereotipamente col plaudite a un pubblico che non esiste - di contro al lettore che il critico immagina retrospettivamente come spettatore! - , e di cui non hanno alcuna idea relativamente a una collocazione di fronte a uno «spettacolo». Un evidente punto di partenza è rappresentato dal caso esemplare di Hrosvita — canonichessa dell'abbazia di Gandersheim, che nel x secolo oppone al Terenzio immorale dei pagani una sua imitazione moralizzata e cristianizzata - e poi dalla cosiddetta commedia elegiaca, che appunto in distici elegiaci alterna narrazione e dialogo.

Di grande interesse l'esempio, peraltro precocissimo, del Babio, un testo francese del XII secolo, che presenta una dichiarazione evidentemente fraintesa da alcuni studiosi: 
Introducit auctor eorum quinque principales personas queque loquens ad se invicem ut coram videretur sermo haberi tanquam a presentibus et ne ambiguitas haberetur que persona cui loquitur. ${ }^{4}$

Si faccia attenzione: le voci dei personaggi si ascoltano attraverso la parola dell'autore, che li introduce a parlare e che li «distingue» (come faranno gli autori dei grandi romanzi più vicini al nostro tempo, soprattutto quelli che Bachtin chiama "polifonici»); un autore in possesso della nozione di modo drammati$c o$ non potrebbe concepire questa ambiguità, né di conseguenza provare a chiarirla. Si tratta, infatti, di un passo che mostra, ad un tempo, la preoccupazione dell'autore di avvicinarsi alla dimensione dell'oralità ma anche la totale assenza dell'idea di una possibile "esecuzione a più voci» in uno spazio fisicamente connotato.

Un'altra questione riguarda la materia del contenuto della commedia «senza teatro» e la sua ricaduta narrativa. Più in generale, infatti, la commedia umanistica — da Petrarca (della cui Philologia Philostrati resta un minuscolo frammento) o senźaltro dal Paulus di Pier Paolo Vergerio- è stata un'esperienza di grande rilievo, proprio nel fatto che un gesto di sostanziale imitazione classica abbia finito col creare oggetti distanti dai modelli che gli autori si erano provati ad imitare. Queste commedie in latino — proprio per l'assenza di coscienza della rappresentazione teatrale — risultano, alla fine, assai più indipendenti dalla commedia classica di quanto non siano le prime restituzioni di testi comici in volgare, destinati alla rappresentazione, nella tradizione rinascimentale italiana, al principio del XVI secolo: commedie, queste, assai più ligie a Plauto e Terenzio e alla fenomenologia drammatica da essi desumibile (a partire dalla definizione spazio-temporale). Il risultato dell'imitazione della commedia umanistica a partire da un vano sforzo imitativo - come ha osservato benissimo Francisco Rico nella prefazione a un testo a questo proposito «mostruosamente» esemplare, La Celestina - finì col «trocar el verso de Terencio por la prosa de la contemporaneidad».5 Ecco dunque il paradosso — solo apparente - di una «rappresentazione della realtà» in assenza di una attualizzazione del modo drammatico. Furono la lateralità e la secondarietà della commedia, insieme alle lacune della conoscenza filologica e archeologica, a permettere ad essa di farsi «el género irremplazable para verter en la literatura de imaginación el interés por la realidad familiar y la curiosidad por la experiencia individual que tan decisamente contribuyeron a moldar el paradigma intelectual de los studia humanitatis» (p. XXIV). Ecco, dunque, un'esperienza variopinta, un género chico, palpitante di quotidianità, dall'immissione nel mondo dell'esperienza amorosa, alla realtà della vita universitaria. Infatti non

4. Gustave CoHEN, La comédie latine en France au XII siècle, Paris: Les Belles Lettres, 1931, II, p. 97.

5. Francisco RiCO, La realidad y el estilo (El humanismo de "La Celestina)», in Fernando de Rojas (y "Antiguo Autor»), La Celestina. Tragicomedia de Calisto y Melibea, edición y estudio de Francisco J. Lobrera y Guillermo Serés, Madrid: Crítica, 2000, p. XV-XLVII: p. XXIII. 
i grandi bastioni della più raffinata erudizione ma le città universitarie italiane furono i luoghi in cui questi testi furono scritti e che fanno ad essi da sfondo ambientativo. L'itinerario che lo studioso traccia brevemente dal frammento petrachesco alla Celestina - il caso più eclatante tra eredità della commedia umanistica latina e novità volgare, col suo immenso successo nel nuovo teatro europeo del XVI secolo (altro caso di "teatralità» in atto o potenza, però in un'età in cui il teatro è ritornato altrove ad esistere come esperienza rappresentativa) - ci sembra esemplare per la comprensione della ricchezza teatrale di un genere che non conobbe e non ebbe come scopo, ignorandola, la realizzazione spettacolare.

\section{III}

L'«atto» o la «potenza» che dir si vogliano sono una conseguenza non tanto di un precorrimento del moderno che deve venire, magari in virtù di una seconda categoria, ancora più gassosa della prima, quella di «laicità». L'evoluzione dal teatro sacro al teatro profano è infatti spesso una comoda invenzione retrospettiva, laddove le letterature nazionali sono state fondate nella disgiunzione dall'eredità classica e nella valorizzazione di autoctoni «medioevi». E l'antico, che sapeva cos'erano il teatro e il modo drammatico in opposizione o rapporto al modo narrativo, che trasmette, di ricaduta in ricaduta, fino alla comprensione ritrovata del moderno, questa eredità, (quella — prima presente ma non compresa - che fa del teatro nel medioevo, come dice benissimo Sandra Pietrini, sostanzialmente una immagine di parole). Molto spesso quello che chiamiamo teatro sacro nasce dalla riconversione - in età che certo propriamente medievale non si può dire- delle forme e dell'esperienza ritrovate dall'antico. Le storie dei teatri nazionali sono piene di esempi di medievalità postuma e inattendibile, dai «misteri» francesi del secondo quattrocento e del primo cinquecento, alle sacre rappresentazioni fiorentine del tempo di Feo Belcari e giù giù, fino alle attestazioni della scena a mansiones medievale dalle immagi$\mathrm{ni}$ - che non mancano in nessun manuale di storia del teatro- della Passion de Valenciennes, che datano al 1577, o l'esempio di sacra rappresentazione testimoniato dalla Rappresentazione di Santa Uliva, che non manca mai dalle antologie del dramma sacro medievale italiano, la cui prima edizione a stampa (e nulla prova che si tratti di un recupero allora archeologico) è del 1568.

Ma non divaghiamo troppo e torniamo all'uso che del Decameron viene fatto quando il senso e l'esperienza del teatro riprendono il loro corso effettivo. Anzitutto il nostro "teatro" o la nostra "teatralità» non sono esattamente quelli che i primi adattatori di novelle decameroniane al teatro hanno cercato nel centonevelle, subito al di qua della «linea moderna». Per «linea moderna» intendo quella che benissimo rappresentano, in prima istanza, i padri fondatori toscani, come Machiavelli e Bibbiena. Dopo l'età repubblicana e savonaroliana, tanto il primo dei vincitori - il futuro cardinal Bibbiena, che compone la Calandria per la festa urbinate del ritorno e trionfo dei Medici del 1513quanto il principale sconfitto, Machiavelli — costretto dagli stessi a volgere il 
viso da un'altra parte e a scrivere cose meno compromettenti, come la Mandragola - , comprendono al bisogno come la loro appartenenza toscana trovasse naturalmente nel centonovelle uno strumento di straordinaria efficacia per spostare l'asse dall'imitazione teatrale della commedia latina (quella degli antighi muorti, dirà Ruzante) rispetto al primato ferrarese, con nuova immissione di beffe e corna e altri ingredienti correlati. Scoprono, insomma, quello che anche noi riteniamo potenzialmente «teatrale» o meglio «teatrabile» nel Decameron.

Di Boccaccio i toscani precursori mostrano tuttavia di aver fatto subito prima un altro, e presto inservibile uso: Bernardo Accolti sceneggia nella Virginia (1494) la novella di Gilletta di Narbona (III 9), Jacopo Nardi nella sua Commedia di Amicizia (ante 1512) quella di Tito e Gisippo (X 8) e nei Due felici rivali quella di Giannole e Minghino (V 5). E altro Boccaccio, diverso da quello del centonovelle, serve alla loro invenzione teatrale. La letteratura critica parla al riguardo di «attardamento», ma forse più che di un ritardo sul fronte della storia - poiché questi autori scoprono il teatro nella sua effetiva destinazione scenica - si tratta qui di osservare una strada tanto innovativa, rispetto anche all'utilizzo novellistico della commedia umanistica, quanto poco fortunata e propizia nella scelta di elementi rispondenti al teatro «rinascente» nel repertorio novellistico e nel suo massimo esemplare. Anche questa è una delle esperienze, e tra le più precoci, della «teatrabilità» del Decameron, storicamente interessante proprio perché lontana dalla nostra sensibilità retrospettiva.

Da un piano di ingredienti (temi, motivi, personaggi e storie, modelli linguistici) proverei però ad allargare lo sguardo, alla ricerca di un più profondo movente o fondamento culturale. Proviamo a cercarlo nella terra di nessuno che si stende tra memoria lacunosa dell'antico e invenzione moderna del teatro. Lidea «medievale» del teatro consiste proprio e principalmente nella incatturabile alterità di un'esperienza dell'antico rispetto alla letteratura in forma dialogata che imita le commedie latine: l'oblio delle sue condizioni fattuali del teatro la determina. ${ }^{6}$ Nel suo commento al De civitate Dei di Agostino, dove si tratta della condanna dei ludi scenici nella città cristiana, Raoul de Presles — che attinge soprattutto al commento latino di Thomas Waleysoffre nei paraggi cronologici di Boccaccio un'idea insieme antica e rinnovata del teatro, immaginandosi una commedia agita; agita almeno fisicamente, nell'estrema brutalità delle azioni dei mimi che si credevano figurare (come racconta Pietro Alighieri, commentando la fatica del padre, che alla comedía si intitola) il contenuto delle parole del poeta o cantor (non certo le parole dell'imitato Terenzio):

Comedie est faite de fais des personnes privees et de puteries de femmes et des hommes et des conchiemens qui se faisoient en telles ribaudies.

6. Perr una fruttuosa riconsiderazione di questi temi cfr. Sandra PIETRINII, Spettacoli e immaginario teatrale nel medioevo, Roma: Bulzoni, 2001. 
E ancora:

ilz se desordonnoient tellement que ilz se despouilloient devant tout le peuple et sans vergoigne venoient iusques aux accolemens et baisiers et au surplus achevoient leur puterie et laidure.

A partire da queste parole, come si vede, si potrebbe addirittura tracciare una doppia linea, nella correlazione e opposizione di scenico ed osceno, particolarmente rilevante per la storia della commedia a partire dalla sua nuova, effettiva, esistenza rappresentativa dalla seconda metà del XV secolo. Una linea che, in negativo, disegna la secolare polemica contro il teatro e che contemporaneamente, in positivo, pone la questione dei limiti di ciò che è rappresentabile sulla scena. Mentre Plauto e Terenzio rientravano in acquisiti canoni letterari, senza generare particolari inquietudini rispetto alla moralità delle loro opere, è come se -in una scissione completa del ruolo del cantor e dei mimi- venisse affidato allo spettacolo perduto incarnare i contenuti proibiti o rimossi.

Se dovessi scegliere un luogo che definisce l'essenza di quella che abbiamo detto "commedia moderna» sceglierei senz'altro quanto Marin Sanudo annota di fronte a una commedia di Ruzante, che egli dice composta tutta «de ficarie et far beco i so mariti». Una definizione che contiene — siamo nel 1525 - ciò che hanno insegnato i formidabili anni che fondano sul Decameron e sul repertorio novellistico la rinascita della commedia, il suo principio del distacco — come direbbe Panofsky — dall'imitazione della commedia classica. Non perché noi siamo così sciocchi da riassumere il centonovelle sotto il segno delle "ficarìe", o eventualmente delle ficarie e delle beffe — quand'anche questi elementi abbiano storicamente determinato la categoria del «boccaccesco»-, ma per l'evidenza dello scarto dalla commedia classica che attraverso essi si gioca: qui di donne, al pari degli uomini, libere e agenti, e non solo di giovani e vecchi maritati invaghiti di fanciulle o di schiave. E ancora, e forse soprattutto, il luogo di Sanudo ci interessa perché fissa l'attenzione sulle spettatrici in sala e sulle loro reazioni davanti alle donne della scena (certamente impersonate da uomini travestiti). Ecco allora che gli «ingredienti» di cui abbiamo detto indicano la rivelazione parallela e inaspettata di un fondamento. Si tratta della realizzazione puntuale dello sguardo che nel Decameron esiste come privilegiato orizzonte di chi narra e di chi ascolta, fin dall'incipit: "Quantunque volte, graziosissime donne...», e ancora, all'altezza della quarta giornata: "Carissime donne...». Un rapporto che non merita solo di essere raccontato nella direzione progressiva della libertà — se non di agire- almeno di leggere o guardare azioni di donne spigliate e pronte. Marin Sanudo legge - in data 9 febbraio 1525 - una protesta negli occhi delle spettatrici di quella commedia: "se agrizavano a quello era ditto per so nome». Ecco, dunque, l'osceno - nel senso letterale del termine - che si rivela direttamente per la reazione scandalizzata, ma a partire dal rifiuto di un'identificazione, delle spettatrici. 


\section{IV}

La virata che ora proverò ad imprimere alla questione potrà sembrare un po' brusca, ma è inevitabile per motivi di tempo, da un piano di discussione di materie o contenuti a un piano di discussione di procedimenti formali. Dall' "agrizzarse» colto da Sanudo nello sguardo delle spettatrici veneziane di fronte all'osceno della commedia, alla storia o alla vita delle forme. Questo perché il modello di scena comica italiana rinascimentale, strada o piazza con edifici, funziona come struttura per esporre direttamente alla vista, rappresentare appunto o figurare una parte della storia, narrandone o facendone supporre un'altra a chi guarda, a proposito di quanto parallelamente all'azione si finge avvenire dietro la scena, e in particolare dentro alle case di cui appaiono le facciate sul palco.

Mentre nella commedia "senza teatro» i luoghi prendono continuamente corpo intorno alle parole dei personaggi e alla loro incerta presenza nello spazio, secondo il principio di un assorbimento della dimensione che nella narrazione è descrizione distaccata nella possibilità che la parola dei personaggi ha direttamente di nominare e "far vedere», viceversa la spazializzazione del genere rappresentativo — nel recupero delle coordinate della drammaturgia antica - ripartisce nettamente questa esperienza. Quello, insomma, che noi troviamo «teatrale» —e che lo è, eventualmente, per altre esperienze e altre forme di significazione rappresentativa- è esattamente quanto nella concreta esperienza storica della rinascita della commedia risulta impossibile nella teatralizzazione della novella.

Gli interni sono dunque in commedia - fin dalla rinascita tardo-quattrocentesca e primo-cinquecentesca italiana - luoghi da cui guardarsi, in cui perfino gli utensili domestici hanno occhi e orecchi. Lo afferma esplicitamente una battuta assai celebre, ed esemplare, all'inizio dei Suppositi di Ludovico Ariosto, che fonda un canone a partire dalla dichiarazione di una circostanza, in sé, irrealistica:

Nessuno appare; sì che esci, Polinesta, ne la via, dove ci potremo vedere intorno, e seremo certe almeno non essere da alcuno altro udite. Credo che in casa sino le lettiere e le casse e li usci abbino li orecchi. (I.1)

Non è dato rintracciare, presumibilmente, un altro luogo in cui si manifesti con tanta chiarezza e brevità il principio convenzionale della necessità del teatro a scena fissa di esterno - liberamente modellato sulla tipologia classicadi far parlare i personaggi al centro del suo spazio di questioni che andrebbero sussurrate nell'angolo più appartato della casa, di esternare in racconto o riferta la vita domestica.

Proviamo a coinvolgere un breve campione, di generica impronta boccaccesca, come si diceva prima. Un campione naturalmente non scelto a caso, perché davvero esemplare relativamente alla questione del rappresentabile e del non rappresentabile che ora abbiamo toccato. Nella Calandria del Bibbiena, anzi al suo centro (III.10), troviamo un celebre dialogo equivoco tra Fes- 
senio (fuor dell'uscio) e Samia (dentro). Si tratta letteralmente di una scena «del dentro e del fuori» (cioè di una relazione tra uno spazio impossibilitato alla visualizzazione ed evocato - ma "in diretta»— dalla voce di una persona che non si vede) e - nello stesso tempo- di una scena dove il dentro e il fuori appartengono alla dimensione, altra, del doppio senso, della significazione retta e letterale e della significazione traversa e metaforica: ciò che è referenzialmente "vero" dal punto di vista esterno (la chiave infilata nella serratura) rappresenta — nello spazio interno negato alla visualizzazione- l'atto sessuale che Samia sta (effettivamente) consumando. Questo non solo per la ripresa di situazioni tipicamente decameroniane, ma anche e soprattutto per la teatralizzazione del procedimento stilistico più diffuso nel Decameron, l'eufemismo, come modalità — come si sa grata a Bembo — di alludere a referenti non degni (osceni, appunto) non nominandoli direttamente: dall'originale dimensione dell'eufemismo novellistico come parola attenuante e argutamente allusiva, a quella teatrale dell'apparenza esterna dell'azione fisicamente compresente ma nascosta:

Fessenio fuor de l'uscio. Samia dentro.

FESSENIO: Tic, toc; tic, toc. Sète sordi? Oh! oh! Tic, toc. Aprite. Oh! oh! Tic, toc. Non udite?

SAMIA: Chi picchia?

FESSENIO: Fessenio tuo. Samia, apri.

SAMIA: Ora.

FESSENIO: Perché non apri?

SAMIA: Io mi alzo per metter la chiave nella toppa.

FESSENIO: Presto, se vuoi.

SAMIA: Non trovo il buco.

FESSENIO: Or escine.

SAMIA: Eh! eh! eimè! non si può ancora.

FESSENIO: Perché?

SAMIA: Il buco è pieno.

FESSENIO: Soffia nella chiave.

SAMIA: Fo meglio.

FESSENIO: Che?

SAMIA: Scuoto quant'io posso.

FESSENIO: Che indugi?

SAMIA: Oooh! Laudato sia il manico della vanga, Fessenio, ché ho fatto el

bisogno e ho tutta unta la chiave perché meglio apri.

FESSENIO: Or apri.

SAMIA: Fatto è. Non senti tu ch'io schiavo? Or entra a tuo piacere.

Sul rincaro di questo principio - e peraltro sulla diretta ripresa proprio di questa scena nella Moscheta - Ruzante fonderà la sua poetica dell'osceno, scandalizzando tra l'altro le nobili spettatrici veneziane, di cui abbiamo detto. Nella Betìa (III vv. 373 ss.) una preziosa didascalia dichiara il significato di una scena ancora più complessa: In questo la mare cerca in caxa la Betìa e dice no la trovando. 
Donna Menega, fuori scena, evoca così, via via, la presenza dei maiali, dei pulcini, e finanche il volo rapace del falco nel cortile retrostante alla casa. Tutte cose che ovviamente lo spettatore non vede. Betìa, intanto, pure dentro la casa, finge di essersi ustionata le gambe con una caldiera d'acqua bollente, rovesciata dai maiali, e di essere entrata per medicarsi la scottatura, mentre, in realtà, sta raccogliendo in fretta un po' di masserizia per fuggire col promesso marito e col promesso amante che la attendono fuori. La madre è quasi indotta a lasciare le sue occupazioni per verificare di persona:

\section{Che fêtu mé a cul buson}

in quel cofano a travasare?

Le parole di Betìa - in un piano, peraltro, dal solo punto di vista del doppio senso più complesso - sono evidentemente false, ma perfettamente traducibili, stavolta, dall'esterno, dove stanno, visibili, Zilio e Nale. All'interpretazione oscena delle false situazioni che non si vedono - el mè andò via la pele e analoghi richiami- si aggiunge la maliziosa degradazione da parte dei due compari delle stesse parole di donna Menega, impegnata a furegare el forno: "...to figiuola per altra man / farà furegar el so forno...", e così via, tanto che la mobilitazione della fantasia sessuale li porta, pezzo per pezzo, ad evocare il corpo reale della contadina: gambe grosse, gran cosse, stazza gagiarda e potente, così da preparare la sua apparizione alla vista del pubblico. Alla fine Betia appare, carica di masserizia, a portare a spalle sulla via uno spazio domestico fatto di povere suppellettili; uno spazio che la commedia moderna si impedisce per sua costituzione di rappresentare, più ancora di quello degli atti sconvenienti. ${ }^{7}$

L'eventuale impressione di un'articolazione scenica «arcaica» o "medievale» — come si usa scrivere - può senz'altro evitarsi ed evitarsi l'idea di una «scena squarciata», capace di mostrare gli interni, soprattutto nel confronto col coevo repertorio della drammaturgia cortigiana di tema mitologico, onde risparmiare restituzioni troppo fantasiose di messinscena. Lo spettatore, infatti, non vede ciò che avviene all'interno dello spazio domestico, perché una parete presente, a differenza della quarta parete "d'aria» dell'esperienza scenografica che verrà, glielo impedisce. Analogamente nelle Nozze di Psiche e Cupidine di Galeotto del Carretto (1499) — autore in posizione precoce anche di una commedia «regolare» come Li sei contenti- una nutrita sequenza di didascalie racconta come agli spettatori non arrivassero, delle scene che si svolgono negli spazi interni, dietro le porte chiuse, che le parole. Per esempio: «Cupido in quello instante essendo in letto cum la sua Psiche parla cum lei stando però sempre la camera serrata e dicegli così». Ma anche quando, più in là, la fanciulla riceve le sorelle, non solo gli spettatori non assistono al bagno e al banchetto ma nemmeno vedono le ancelle chiamate ad allietare quel tempo d'attesa col canto: "Queste Ancille non vedute cantano la subsequente canzonetta». Si tratta di indu-

7. Per un'analisi più dettagliata rinvio al mio Il villano in scena. Altri saggi su Ruzante, Padova: Esedra, 2006, p. 53-74. 
bitabili testimonianze di istruzioni di scenificazione di un testo che vanno peraltro nella direzione opposta - torneremo più in là su questo punto- dell'idea diffusa di una pratica "arcaica" di aprire a piacimento le case di commedia per mostrare il loro interno quando l'azione lo richiedesse.

L'osceno - quello che non si può rappresentare- non è però secondo questa funzionalizzazione solo ciò che può essere raccontato ma non mostrato in quanto contenuto non lecito, ma anche ciò che statutariamente appartiene alla dimensione domestica occultata, ed è semplicemente «fuori scena». Non solo gli amplessi o gli atti sessuali, veri o presunti, ma anche le suppellettili domestiche e le umili occupazioni che contraddistinguono quello spazio. L'altra dimensione che la commedia può solo raccontare e non agire, a partire dal racconto ariostesco, negli stessi Suppositi, riguarda, in uno dei pochi cenni in questa direzione, per esempio la mensa del vecchio Cleandro che presenta, per sette persone, compreso il gatto, «uno luccetto d'una libra e mezzo, e una pentoletta di ceci e venti sparagi». Anzi, si potrebbe dire, che mentre le parole sporche e disoneste — come diceva ancora Sanudo- possono riempire la scena anche come parodia trivializzata di ciò che dentro è semplice azione "domestica» (Betia che fa fagotto e sua madre che fa le faccende di casa), è invece proprio della realtà materiale, o creaturale, il non potere essere mostrata sulla scena.

Ciò avviene solo eccezionalmente in termini di teatralizzazione diretta nel panorama della drammaturgia italiana cinquecentesca. In un appartato capolavoro come La Veniexiana, commedia che allarga la ristretta unità del "giorno teatrale» e alterna sorprendentemente interni ed esterni, non a caso le parole che aprivano I suppositi sono letteralmente ribaltate, quasi a sottolineare una simile opzione (ma altresì a mostrare che l'autore conosce le modalità statutarie di scenificazione):

Messer Iulio caro, el se suol dir che el xe matiera parlar cusì a la scoverta, perché i venti ha orechie e occhi. Vegné dentro e me alegraré un puoco a véderve a la luse. (IV. 73)

Non entrerò qui nella complessa questione relativa all'esecuzione della Veniexiana, limitandomi però a raccogliere il cenno a una destinazione scenica che il Prologo esibisce e che, guarda caso, affronta proprio la questione della rappresentabilità dell'osceno, come publicazione di quanto sarebbe da passar sotto silenzio:

Tutti, vi prego, prestate orechie e in alcuna parte non vi turbate, se quello, che da sé è da passar sotto silenzio, oggi d'i nostri mimi senza vergogna serà publicato: imperò che, dobiando esser ben edocti de la proprietà de amore, è necessario che tuti soi effecti distinctamente cognosciate. (\$ 6)

La Veniexiana "mostra di più" proprio in quanto testo più fortemente condizionato dalle forme narrative, proprio perché essa tenta una scenificazione di una historia oltre i limiti convenzionali della fabula e della comedia, come affer- 
ma il suo sottotitolo. Entrare nella camera da letto della vedova Angela significa anche, ad un tempo, poter evocare in presenza - almeno in una presenza simbolica, come quella di ogni teatro dello spazio «significato» dalla parolagli oggetti e gli arredi della vita quotidiana, dal letto, allo scaldino, agli incensi bruciati per profumare la stanza. Le suppellettili e gli oggetti della vita quotidiana sono qui nominati non perché collocati altrove, ma perché disegnino uno spazio — quand'anche siano evocati, com'è probabile, solo dalla parolaintorno ai personaggi. La Veniexiana è un testo ai nostri occhi più "teatrale» in quanto, in realtà, più prossimo all'orizzonte della narrazione.

\section{V}

Mi concentrerò brevemente su un esempio ragguardevole di «teatralizzazione» di Boccaccio. Ho scelto senza indugio Il Filosofo di Pietro Aretino (1544), per più ragioni. Ne elenco almeno tre: 1) si tratta della sceneggiatura di una intera novella decameroniana (quella, celeberrima, di Andreuccio da Perugia: II 5) e non della semplice ripresa di uno o più elementi sparsi; 2) si tratta di un esempio che mostra un trattamento in controtendenza rispetto alle nostre attese di «teatralità» retrospettiva, nell'assoluta divaricazione dalla lingua e dalle strutture di parola e gesto nello spazio-tempo della novella che ci attenderemmo realizzate; 3) infine si tratta di un esempio complesso, benché convenzionale, di impiego dello spazio della scena fissa in rapporto alle necessità ambientative della novella.

Che si tratti, infine e più generalmente, di un caso ragguardevole di imitazione era perfettamente evidente allo stesso Aretino, che non a caso chiama direttamente il suo Andreuccio col nome di Boccaccio. Non credo perché la commedia rilevi le vaghe pendenze autobiografiche della novella, giacché lo straordinario spaccato di realtà napoletana che essa offre resta del tutto lettera morta nella teatralizzazione. Il nome dell'autore sostituisce quello del personaggio come deliberato riconoscimento di dipendenza, esplicitamente sottolineato, per mostrare la direzione bizzarra del trapianto da un genere e da un modo a un altro e la convenzionalità che fonda il grado altrimenti bizzarro della realizzazione.

La trama è innestata - come ormai di consuetudine a questa altezza cronologica - in una tessitura a duplice filo: la vicenda di Andreuccio è alternata a quella di Plataristotile — che intitola come «filosofo» la commedia—, che pure possiede late pendenze decameroniane (la novella di Arriguccio Berlinghieri, VII 8). Sul versante che ho indicato al punto due delle tre ragioni essenziali di scelta, è estremamente significativo l'intreccio di una spedita sequenza d'azione, che riguarda esclusivamente gli snodi essenziali della trama di Andreuccio, in una commedia dominata dall'espansione verbale senza posa, non solo nelle prevedibili pappolate del filosofo imbecille ma anche in sede dello scambio verbale tra i personaggi. Questo svolgimento, ripeto, contraddice vistosamente le attese più prevedibili della derivazione decameroniana, al pari del sacrificio, in una novella tanto famosa per questo, di ogni colore locale: non 
la Napoli notturna e malfamata dell'originale ma nemmeno un altro ambiente equivalente, per esempio la Venezia in cui l'autore opera, tanto che la solita pattuglia di sbirri parla qui un veneziano d'intonazione stereotipa rispetto al ruolo evidentemente codificato degli zaffi, che però non ha alcuna pretesa di rinvio a una qualche realtà. Bisognerebbe certo meditare di più su questa astrazione, che è un carattere deliberato dell'operazione aretiniana e che non è, come si può credere, un semplice difetto di elaborazione.

Si veda, per esempio, come Aretino rinunci ad alcuni personaggi che non possono non colpire la fantasia di qualsiasi lettore, anche senza particolare mandato di «spettatore». Boccaccio esce infardato sulla strada dopo il crollo dell'asse del cesso che avrebbe dovuto ucciderlo e quindi bussa alla porta della casa della finta sorella, e appare alla finestra Cacciadiavoli ruffiano (III.7-9). Aretino rinuncia ad ogni dilatazione di una scena così, in poten$\mathrm{za}$, teatrale, sopprimendo le presenze circostante del vicinato, e facendo pronunciare al personaggio che esce alla finestra una sola battuta, ma con totale distanziamento dall'impronta di viva oralità dell'originale. Eccole in paragone:

Io non so a che io mi tegno che io non vegno là giù, e deati tante bastonate quante io ti vegga muovere, asino fastidioso e ebriaco che tu dei essere, che questa notte non ci lascerai dormire persona.

Che stregaria di maliamento d'anima dannata è istanotte cotesta tua, vigliacco? stupisco del non sapere il perché m'indugio a correrte adosso gettandoti la testa mezzo miglio lontan dal busto.

È come se una formulazione diretta, e appena coperta dalla patina letteraria, fosse, anche in una breve presa di parola, impossibile, e come se ogni enunciato dovesse essere traslitterato in una resa artificiale e straniata: può un ruffianaccio buttato giù a piena notte dal letto esordire con un stregaria di maliamento, può minacciare qualcuno chiedendosi di stupire di non sapere? Infatti questo figuro nemmeno ha necessità di sbadigliare e stropicciarsi parlando come fa - si direbbe «teatralmente» - l'omone con la barba nera e folta che apostrofa Andreuccio. Potremmo fare molti altri esempi, ma questo basta a dire come la commedia aretiniana divarichi la distanza tra cosa e parola, secondo un'attitudine straniata e allucinata. È come se i personaggi fossero parlati da altri, mentre il toscano colorito e gergalmente ispessito che essi parlano risulta altrettanto e più difficile delle tante lingue contraffatte che in quello stesso momento si usavano sulla scena veneziana: questa persa nello spazio del proprio lambiccamento, quelle pur sempre contraffazioni della realtà e presumibilmente appoggiate alla comunicazione mimica.

Per contro il «teatro» è per Aretino, oltre la trama di parole, nella gratuità dell'azione: un vero e proprio tocco d'arte è nell'apparizione alla finestra appena prima dell'ingresso nel cesso di cui sono state manomesse le assi, di Boccaccio: egli si affaccia solo a vedere cosa abbiano da urlare i birri che passano in quel momento per la via. Certo, l'apparizione serve a localizzare Boccaccio 
dentro la casa, a premessa della sua prossima caduta (che ovviamente non si vede: il vicolo-latrina sta solo idealmente nello spazio dietro alle case, impedito alla vista), e insieme a far sfilare una prima volta la pattuglia dei vigilantes, ma sulla funzionalità vince prepotentemente quell'impronta di gratuità che aveva fatto nella Cortegiana sfilare in scena dei personaggi intenti solo a passeggiare chiacchierando.

La porzione più interessante della teatralizzazione aretiniana è tuttavia nelle scene seguenti del terzo atto, fino al principio del quinto e riguarda le successive peripezie di Boccaccio, dalla lavatura nel pozzo al furto dell'anello del vescovo sepolto di fresco. Si tratta di una situazione, infatti, che richiede un'azione ambientata all'interno della chiesa (che qui è intitolata alla solita Santa Nafissa, martire, vergine non dico già).

È sempre difficile e pericoloso tentare di immaginare la scenificazione di un testo, tuttavia in questo caso è lecito supporre quanto segue: la tomba del vescovo è situata sul pavimento, e non è più un'arca «di marmo e molto grande» come nell'originale, ma un sepolcro e provvidenzialmente collocato vicino alla porta ("ch'è apunto in su l'entrata»), dettaglio del tutto assente nell'originale; in secondo luogo, le battute della commedia sono molto dettagliate relativamente alla scassinatura della porta, ciò che nella novella non importa nemmeno riferire («n’andarono alla chiesa maggiore, e in quella assai leggiermente entrarono»). Le battute dei personaggi aretiniani ci ragguagliano, invece, sul fatto che la porta deve essere aperta, evidentemente per permettere agli spettatori di vedere l'azione della calatura di Boccaccio nel sepolcro, collocato come abbiamo visto nelle immediate vicinanze dell'entrata. Quando arriva il secondo gruppo di ladri, dopo la chiusura del poveretto dentro il sepolcro e la fuga dei suoi compagni (qui i secondi ladri si chiamano Mezzoprete, Sfratato e Chietino, con ovvia indicazione del loro rango), mentre la novella nulla dice sul loro ingresso, la commedia sottolinea il dettaglio della porta già aperta. I personaggi non rilevano il fatto che qualcuno deve averli preceduti, ma la comodità ad altro fine della circostanza:

\section{Ella si sta così per iscemarci fatiga. (IV.12.16)}

Tra il quarto e il quinto atto la continuità scenica è assoluta. Dopo la fuga del secondo gruppo di ladri, che conclude il quarto, si sente la voce di Boccaccio, evidentemente a scena vuota, che descrive i suoi movimenti per uscire dalla fossa con la solita attitudine straniata ( $\mathrm{Ci}$ salirò pure. Isbalzami in sù, persona; perdonami, ginocchio, $s$ 'io ti stroppio col premermiti tutto sopra», ecc.), e poco dopo appare alla vista, e riguadagna la strada, uscendo dalla chiesa.

Il Filosofo richiede, dunque, una visualizzazione parziale di una porzione di un interno ottenuta semplicemente, e non senza ingegnosità, col semplice artificio di una porta aperta. 


\section{VI}

Se volessimo osservare le modalità di visualizzazione della vicenda di Andreuccio nelle miniature e nelle illustrazioni decameroniane vedremmo, ovviamente, che il criterio di gran lunga prevalente per figurare in immagine ciò che si svolge dentro la chiesa - ma anche nello spazio tra le case della latrina in cui Andreuccio atterra salvandosi la vita— è nella «squarciatura» o abolizione delle pareti divisiorie, secondo un tipico procedimento dell'illustrazione narrativa. Ne riporto due, a titolo d'esempio e di confronto a partire dall'evidenza di un unico impianto, da due manoscritti francesi rispettivamente del primo e del secondo quarto del XV secolo [fig. 1]. ${ }^{8}$ Andreuccio appare in entrambe le illustrazioni, a sviluppo multiplo o sequenziale, due volte: la prima nella latrina tra le case, la seconda mentre si cala nella tomba (anche qui collocata sul pavimento). Nella prima egli è praticamente nudo, nella seconda risulta in sottoveste; la prima mostra il poco raccomandabile uomo che si affaccia alla finestra e la seconda lo sostituisce con due generiche presenze, nell'evidente perdita del nesso narrativo; la seconda, tuttavia, estende significativamente il principio della «apertura» degli interni, dichiarando l'artificio che lo regge: una porzioncina del muretto divisorio si mostra davanti ad Andreuccio accovacciato nella latrina, mentre addirittura è aggiunto il particolare dell'interno del cesso, sospeso tra le due case, grazie alla soppressione di alcune assi della parete che ne celerebbero la vista.

Ho riportato queste immagini solo in rapporto al fatto di avere appena messo in campo una teatralizzaione della novella di Andreuccio che va in direzione esattamente contraria dei luoghi comuni di quell'invenzione scenografica retrospettiva che usa trarre indebite testimonianze dal linguaggio delle immagini. Ovviamente il procedimento della visualizzaione per sequenza multipla (solitamente a scansione binaria), che presenta più volte il personaggio o i personaggi principali di una novella, si appoggia naturalmente alla convenzione - tipica, del resto, della pittura narrativa - della «scena squarciata». Si tratta di un procedimento largamente diffuso, che per quanto riguarda la tradizione decameroniana si ritrova fin da i manoscritti più antichi. Lo stesso prezioso codice da cui abbiamo tratto la seconda immagine ora commentata, ci presenta per la visualizzazione dell'ottava novella della seconda giornata un omino, che sta per il conte d'Anguersa, addirittura in atto di uscire dallo spazio dell'ideale quarta parete. Non siamo, ovviamente, a teatro, e infatti il gesto sottolinea piuttosto l'attitudine di andare del personaggio, dopo le parole della nuora del re di Francia [fig. 2].

Veniamo anche qui a qualche indicazione di portata generale. Ho sempre provato profonda antipatia per l'ipotesi o il «metodo» di Pierre Francastel, di vedere il "teatro" nella pittura. Molto diffuse sono le fantasie che si applicano a casi ragguardevoli della pittura del rinascimento, in cerca di un riflesso sce

8. Biblioteca Apostolica Vaticana, Palatino latino 1989 A e Bibliothèque de l'Arsenal, 5070 B: cfr. Vittore BRANCA (a cura di) Boccaccio visualizzato, Narrare per parole e per immagini fra Medioevo e Rinascimento, Torino: Einaudi, 1999, III, schede 83 e 85. 

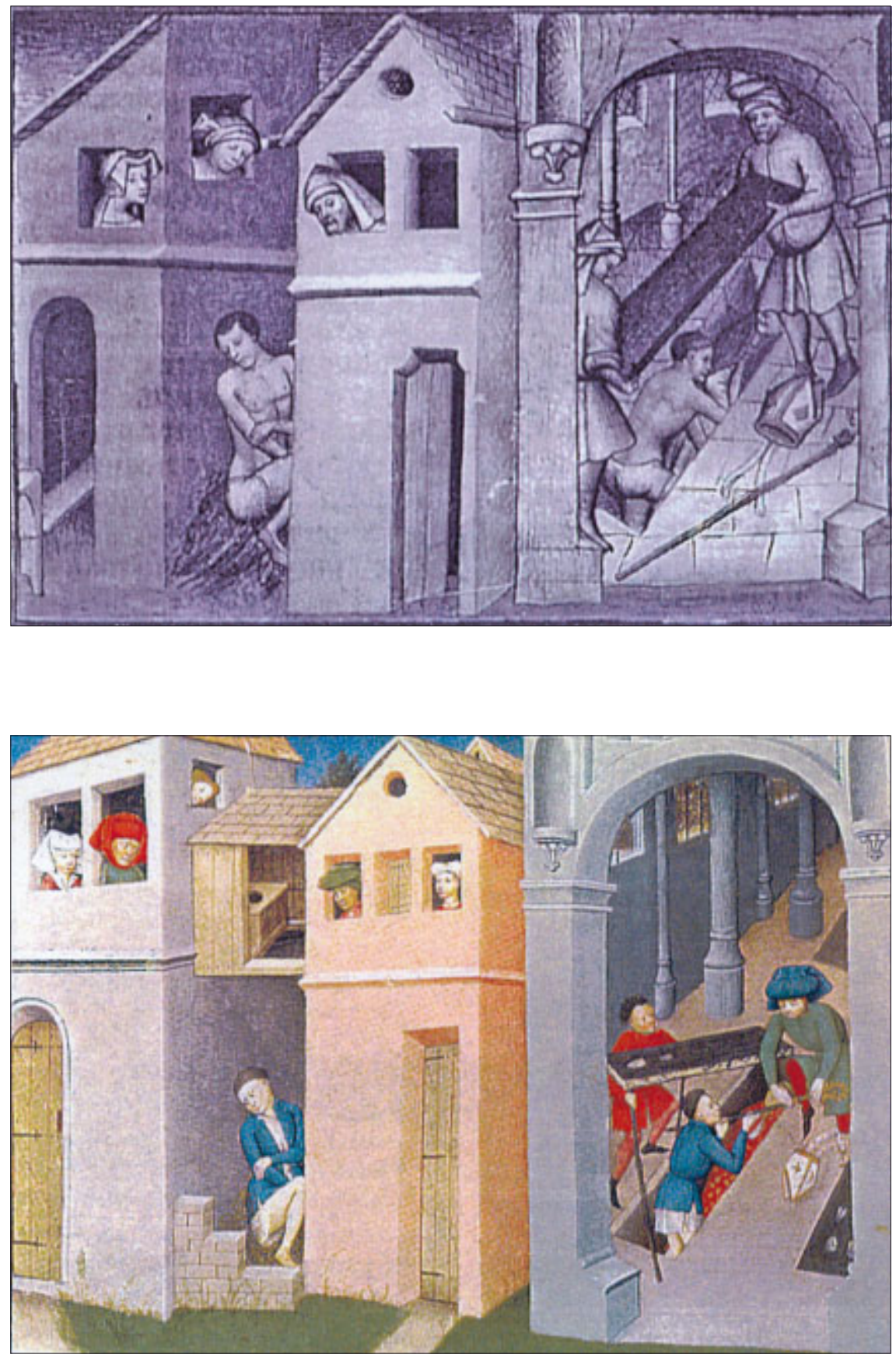

Fig. 1 


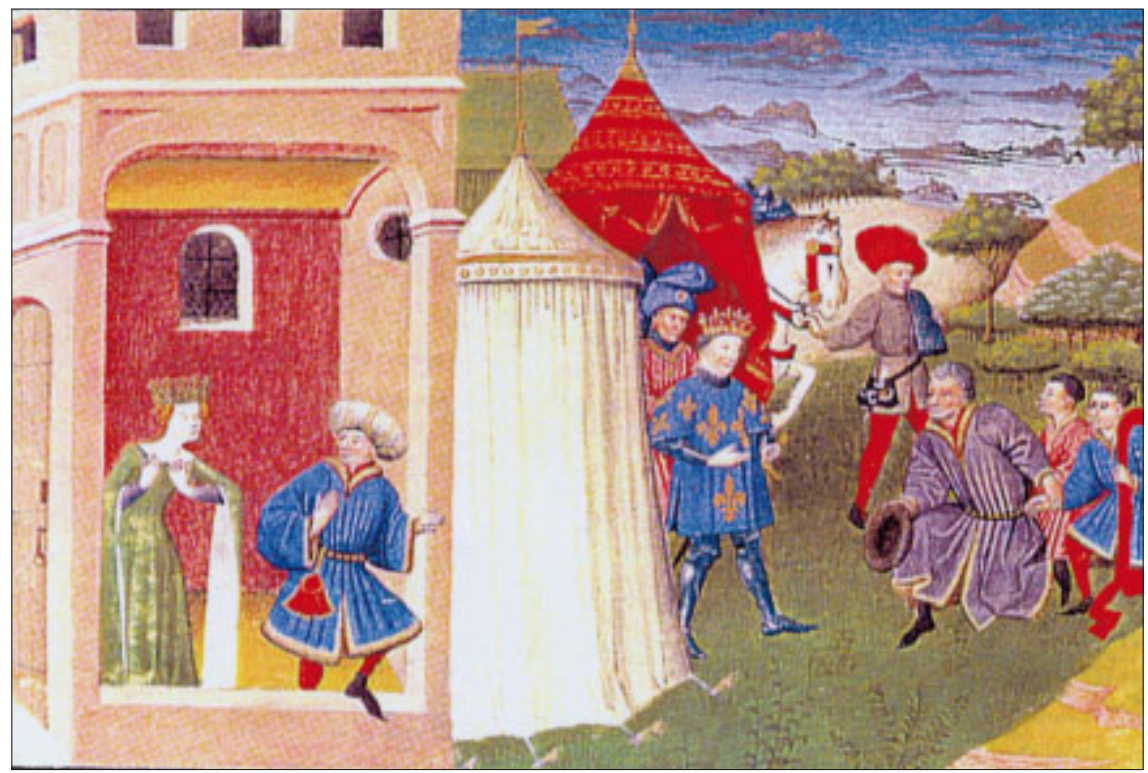

Fig. 2

nico che non ha alcuna giustificazione oggettiva negli elementi referenziali del dipinto. Esemplari quelle che riguardano il ciclo di Sant'Orsola di Vittor Carpaccio, letto come se alcuni dei suoi teleri mostrassero delle scene della storia della Santa come prese da una sacra rappresentazione ad essa dedicata (mentre il «teatro» di Carpaccio, presentissimo nei teleri, è nel rituale dell'ambasceria). Per esempio quella della scena a sinistra del dipinto intitolato l'Arrivo degli ambasciatori. Anche qui il re di Bretagna appare due volte: prima nella scena centrale sotto alla loggia, in trono, a ricevere dagli ambasciatori del re d'Inghilterra la richiesta della mano della principessa Orsola; quindi all'interno di una stanza, seduto accanto al letto, pensieroso, mentre ascolta le condizioni che sua figlia Orsola pone per sposare il principe inglese [fig. 3].

Ludovico Zorzi ha visto qui addirittura una riproduzione non di una stanza, ma di un "palcoscenico che mostra una stanza». Si tratta - da parte di un interprete di straordinaria cultura e intelligenza - di un francastelismo del tutto irricevibile, che fonda una lunga analisi che giunge addirittura a fantasticare sul personaggio della vecchia seduta sui gradini esterni alla «scatola dell'ambientepalcoscenico», al di qua di una parte che non c'è, come "un personaggio che attende di fare il suo ingresso in scena». ${ }^{9}$ Non sarà che la vecchia, balia o nutrice, se ne sta fuori, oltre che per significare le implicazioni dello spazio pittorico nella «realtà» referenziale, proprio perché la conversazione che si svolge all'in-

9. Ludovico ZorZI, Carpaccio e la rappresentazione di Sant'Orsola. Ricerche sulla visualità dello spettacolo nel Quattrocento, Torino: Einaudi, 1988, p. 30 ss. 


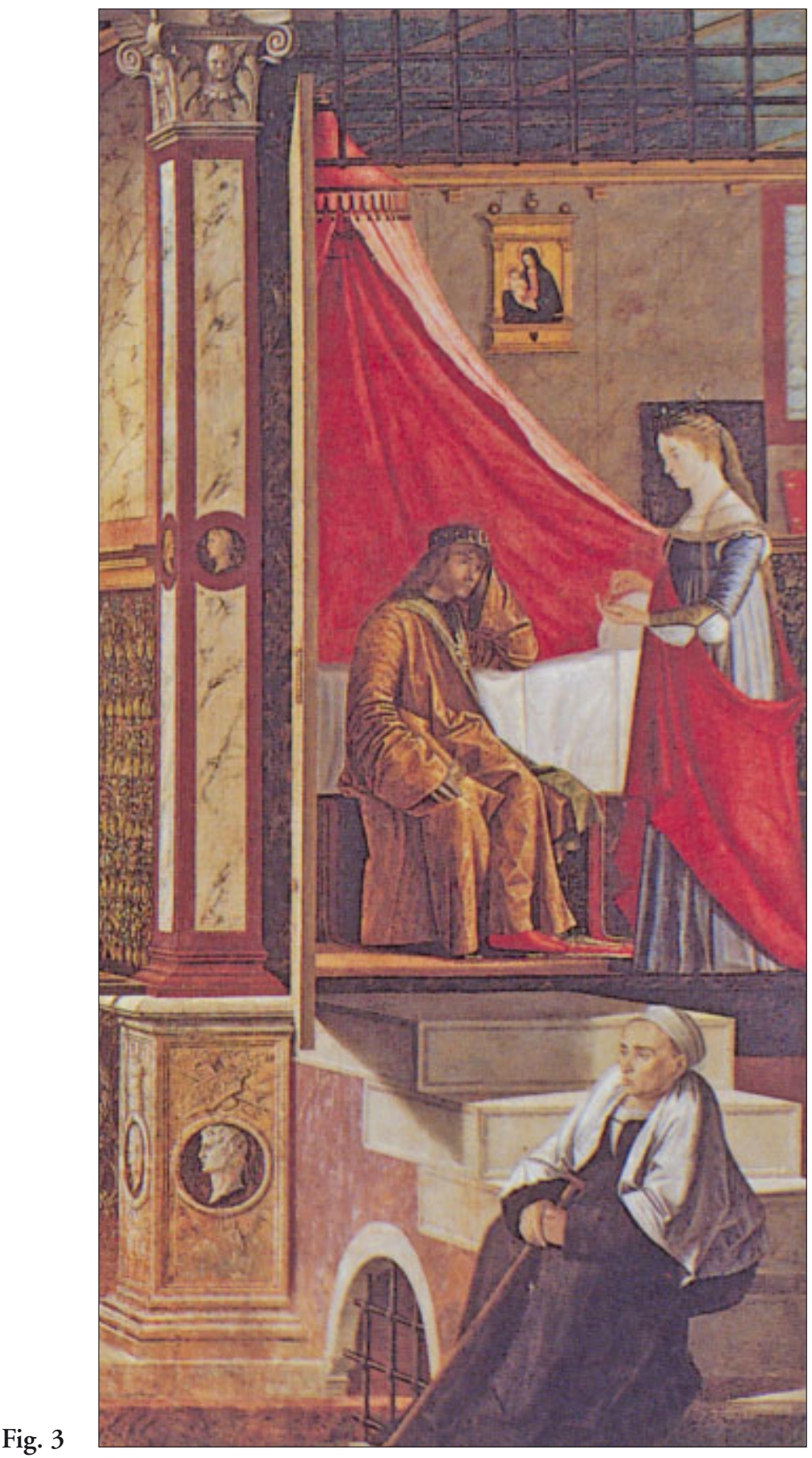


terno della stanza è esclusivamente "privata»? Non ho alcun dubbio che si tratti, dunque, di un esempio eloquente di «teatralizzazione» come invenzione o proiezione retrospettiva del teatro, in un senso diverso ma certo parallelo a quello delle istanze mimiche e drammatiche supposte dentro alla dimensione narrativa, anziché dentro la «realtà» della fabula, da cui siamo partiti. Sul telero sono tornati, in questa prospettiva e con ben altra fantasia (parlando dei soliti «luoghi deputati» e delle «scene squarciate»), in seguito altri studiosi, in pagine che non abbiamo qui il tempo, né in fondo la necessità, di analizzare.

Mi soffermerei solo sulla lettura - nella prima parte di un contributo che contiene nella seconda un'eccellente analisi dedicata alla questione degli espacios interiores nella comedia urbana spagnola del XVII secolo- che mi sembra particolarmente fuori strada, e che mostra i danni di una propagazione in altro ambito disciplinare di ipotesi indimostrate che solo la quantità di riprese finisce col rendere dati certi. ${ }^{10}$ Stefano Arata può definire quella della scena aperta come una modalità scenografica un tempo praticata dal teatro italiano, già in decadenza e vista come arcaica negli anni in cui Leone de' Sommi ne parla, nella seconda metà del XVI secolo, nei suoi Dialoghi in materia di rappresentazioni sceniche (1556) e che poi verrebbe recuperata dal teatro spagnolo. Non può non impressionare come all'atto di citare un lungo brano di questo testo si ignori, di fatto, quanto esso espressamente afferma e si dia credito viceversa a ipotesi che ne contraddicono il significato. Non sto, naturalmente, affermando che Leone de' Sommi non potesse essere poco informato, criticando come moderno e di provenienza spagnola un procedimento viceversa ben noto in Italia e arcaico, ma che tutto ciò dovrebbe essere provato altrimenti. Fino a prova contraria è lecito credere a Leone de' Sommi.

Cosa prova che la scena aperta "procede de las artes visuales» e si incontri nel teatro italiano alla fine del XIV secolo? Ovviamente la lettura di Zorzi del telero carpaccesco che "reproduce las técnicas de puesta en escena del teatro religioso de la epoca» e una testimonianza ferrarese - tratta sempre dal libro di Zorzi- che in realtà descrive le casette con camino della notissima città ferraese, su cui possediamo decine di attestazioni (una seconda testimonianza, che Zorzi ricava da D'Ancona, si limita a menzionare la presenza di un letto in un tableau vivant piemontese).

Ci sono testi - come abbiamo visto con la Veniexiana - che pongono direttamente la questione della loro rappresentabilità (anche nei limiti della sola escogitazione mentale della rappresentazione da parte dell'autore). Certo la pongono per un più complesso rapporto della teatralizzazione dello spazio e del tempo del racconto (della novella) rispetto alle forme convenzionali della scenificazione comica (la commedia anonima è del 1535, di data troppo avanzata e troppo piena di memorie di drammaturgia regolare per essere pensata

10. Stefano Arata, «Casa de muñecas: el descubrimiento de los interiores y la comedia urbana en la época de Lope de Vega», in F. CAZAL, C. GonzÀles, M. Vitse, (a cura di), Homenaje a Frédéric Serralta. El espacio y sus representaciones en el teatro español del Siglo de Oro, Universidad de Navarra, 2002, p. 91-111. 
come un prodotto arcaico, ammesso poi che siffatti prodotti arcaici siano mai esistiti concretamente). Forse la Veniexiana - me ne sono occupato altroverisolveva la sua alterità spaziale e temporale, per nulla "arcaica» $\mathrm{O}$ "medievale», in una cultura dello spazio significato, non in una cultura della figurazione dell'historia come scenografia, retrospettivamente immaginata a partire dalle consuetudini della rappresentazione pittorica dello spazio e del tempo «narrativi», a partire dall'indebita mossa di immaginare il teatro come "scenografia».

L'immaginazione retrospettiva degli studiosi ha, del resto, spesso interpretato le illustrazioni di servizio dell'età umanistica come riferimenti diretti alla realtà delle recite contemporanee delle commedie latine. Si pensi alla fortuna - pressoché in ogni libro di storia del teatro- delle illustrazioni che accompagnano le edizioni di Terenzio sullo scorcio del Quattrocento. Non c'è da stupirsi, dunque, che anche Arata metta in campo come fededegna pezza d'appoggio le celeberrime vignette dell'edizione terenziana di Jacob Trechsel (Lyon, 1493), mentre non sembra plausibile che egli rinvii a tal fine a un importante saggio che ha come suo merito quello di avere vanificato con solide prove questa vulgata. ${ }^{11}$ Gli autori affermano, infatti, che le vignette dell'edizione lionese - poi riprese da altre edizioni terenziane — non mostrano già la maniera in cui si rappresentava Terenzio, o altro, alla fine del Quattrocento, ma —al di fuori di ogni rispecchiamento di realtà spettacolare- la maniera in cui l'avanguardia dell'applicazione umanistica immaginava ipoteticamente la realtà del teatro antico. Si tratta, anzi, di un vero e proprio "commento per figure», ideato dall'umanista fiammingo Josse Bade van Assche (alla latina Jodocus Ascensius Badius), che non si può capire se non si legge parallelamente il commento per parole che lo stesso ha composto.

Una vignetta relativa all' Andria mostra, per esempio, il proscenio come un palco di legno, che presenta nel fondo quattro porte chiuse da tende (la quarta anzi aperta e che mostra una finestra), riprendendo le idee elaborate dai primi umanisti sulla forma della frons scenae antica e delle sue porte, e ai lati due altarini (con le statue di Phoebus e Liber), che procedono indubbiamente dalla nozione della presenza dell'ara nella scena comica classica [fig.4]. Queste tendine, peraltro, sono immaginate dall'umanista fiammingo solo come chiusura delle porte della scena, visto anche che i testi della commedia latina non pongono nessuna necessità di scene di interno. E quello che la vignetta mostra non è l'interno della casa di scena, ma la dimensione retrostante allo spazio della scena. Per quanto riguarda la disposizione narrativa, si noti la sequenza che mostra due volte — in due momenti successivi- Simone e Sosia (Sosia avvicina Simone e, quindi, gli parla a capo scoperto; Davo e Dromone sono impegnati in fatiche manuali). Qui la frons scenae è —appunto- costituita da porte chiuse da tende allineate (la cosiddetta tipologia della Badezellenbühne, «scena a cabine da bagno») che identificano le "case» dei personaggi

11. Thomas Edward LaWrenson; Helen Purkis, «Les éditions illustrées de Terence dans l'histoire du théâtre», in Jacques JACQUOT (a cura di), Le lieu théatral à la Renaissance Paris: C.N.R.S., 1964, p. 1-23. 


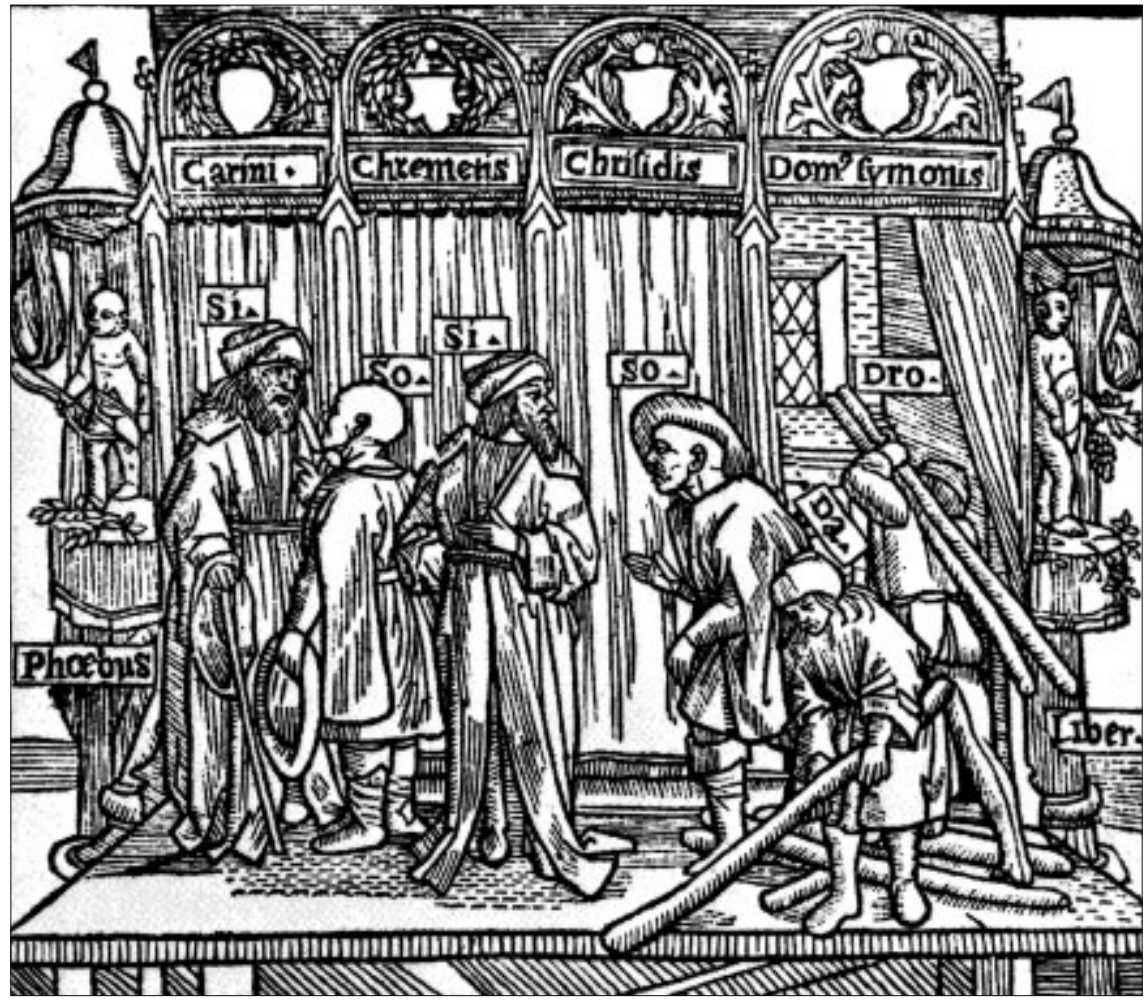

Fig. 4

(quella di Cremete non sarebbe, peraltro, prevista «in scena»). Le illustrazione del Terenzio di Lione sono una testimonianza irripetibile - per densità e rilievo del progetto del «commento» che le fonda- di come l'immaginazione umanistica pensasse il teatro degli antichi e ne offrisse una restituzionevisualizzazione secondo gli schemi dell'illustrazione narrativa, quella usa appunto a distendere in articolazione di sequenza visiva il continuum saliente della narrazione. È evidente come la tradizione della visualizzazione del Decameron — per il piacere di chi legge ma altresì per consentirgli di ricordare leggendo chi agisce nel testo- offre al proposito un versante eccezionalmente pertinente per la comprensione delle regole del gioco. La questione del rapporto tra visualizzazione teatrale e visualizzazione narrativa nell'illustrazione terenziana è, peraltro, un terreno enormemente complesso. Citerò qui un solo esempio, quello del Vaticano latino 3305, un codice realizzato nella Francia del Sud nel XII secolo, a una data evidentemente troppo precoce perché si possa supporre possibile al miniatore un riferimento a una qualche esperienza rappresentativa. Nelle immagini che accompagnano l'Andria più volte ricorre l'espediente della casetta "aperta» per designare le occupazioni da cui Sosia è distratto. Che la 


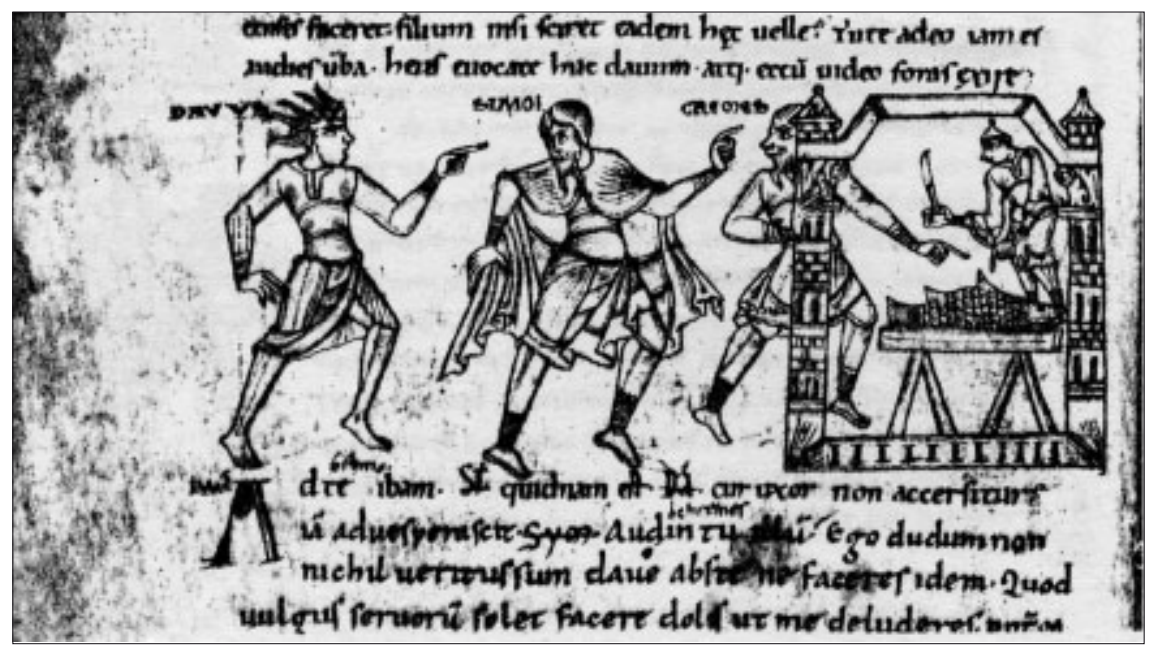

Fig. 5

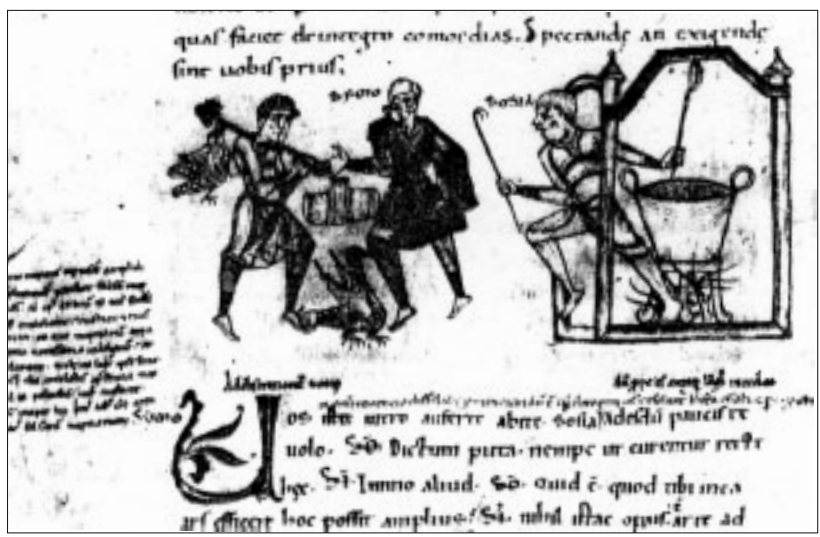

Fig. 6

rappresentazione dell'interno domestico — con tanto di pentola che bolle sul fuoco- sia qui un espediente puramente narrativo è dimostrato, se mai ce ne fosse bisogno, dal fatto che le illustrazioni si riferiscono anche a parti della vicenda che appartengono al racconto dei personaggi e che non si svolgono sulla scena [fig. 5-6]. ${ }^{12}$

Ma torniamo ad Arata e al suo punto d'approdo, con la proposta, pur con qualche cautela, che le illustrazioni dell'edizione della Celestina apparsa a Bur-

12. Per un'analisi delle illustrazioni e per un quadro bibliografico di riferimento cfr. David $\mathrm{H}$. WRIGHT, «The Forgotten Early Romanesque Illustrations of Terence in Vat. lat. 3305», in Zeitschrift für Kunstgeschichte, 56, 1993, p. 183-206. 

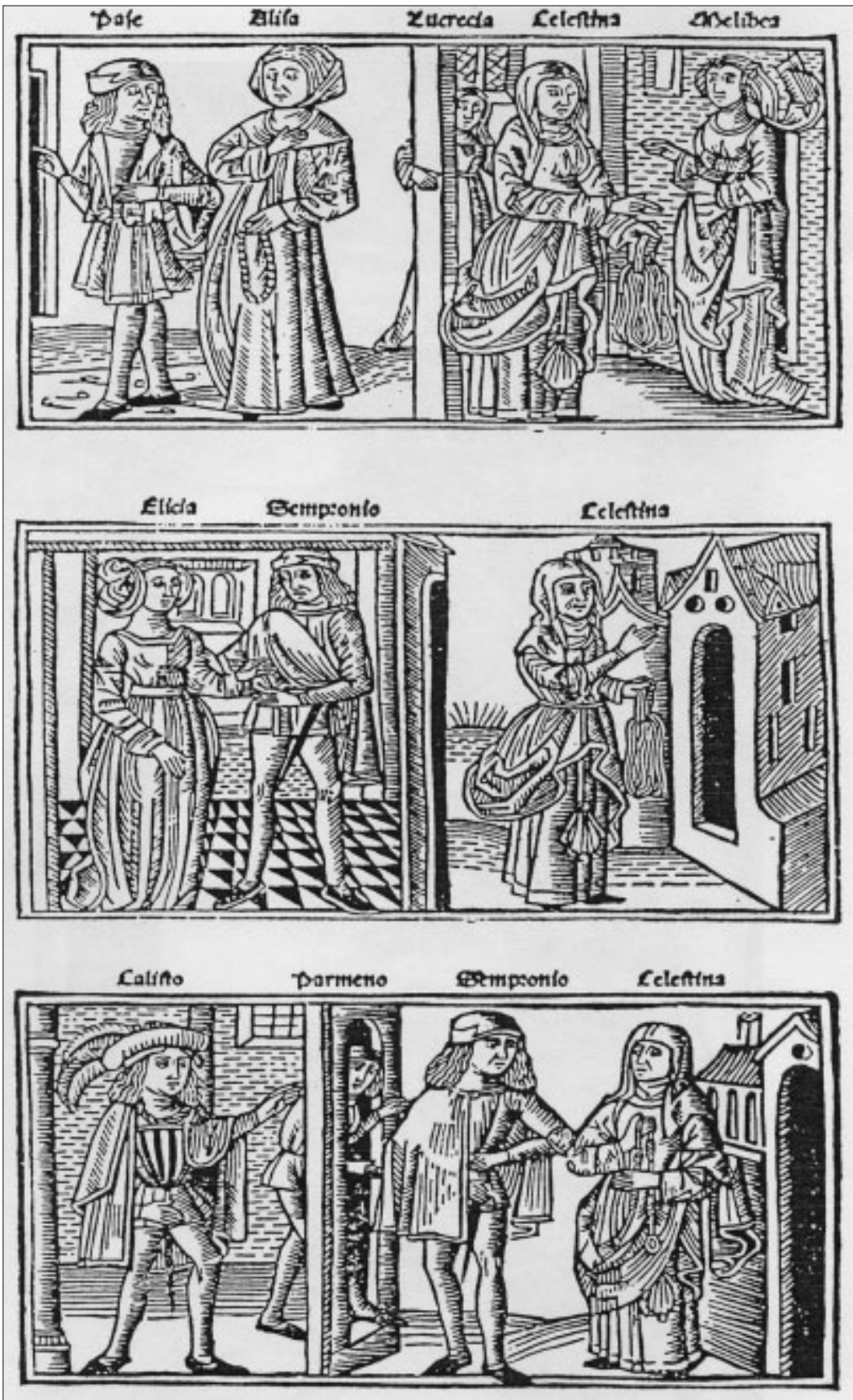

Fig. 7 


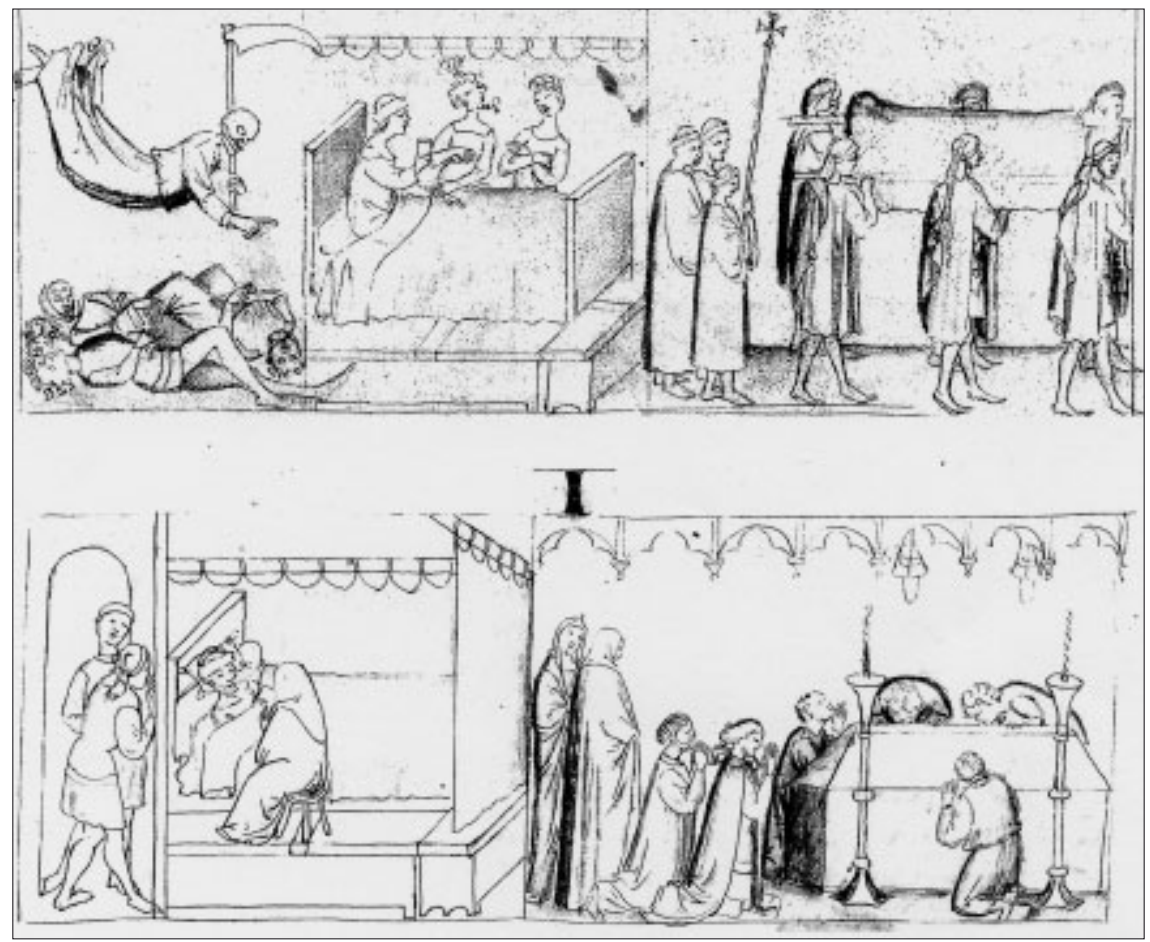

Fig. 8

gos nel 1499 richiamino l'impiego teatrale della scena aperta, anche se la testimonianza non sarebbe dotata dell'evidenza che si coglie nel dipinto di Carpaccio [fig. 7]. Che una coeva edizione apparsa nella stessa città —l'Historia de los nobles caballeros Oliveros de Castilla y Artús d'Algarve- non ricorra nelle sue vignette che illustrano scene d'interno al procedimento della scena aperta, permette però allo studioso di concludere con sicurezza che una tale tecnica era evidentemente «relacionada sólo con el espacio teatral», chiudendo il cerchio dell'ipotesi favorevolmente alla precedente assunzione. È evidente, ed economico, viceversa pensare che relazioni diverse tra modalità di visualizzazione pittorica e narrazione escludano, fino a prova contraria, il teatro.

Attendiamo una testimonianza concreta nel campo della storia dello spettacolo per spostare verso questa l'immenso repertorio della figurazione narrativa che trova nelle centinaia di scene aperte della tradizione di visualizzazione sulla pagina delle novelle del Decameron una delle sue linee più evidenti e rappresentative, che fonda anche, tra parentesi, la tipologia a cui si ispira la Celestina di Burgos; quanto ai nobles caballeros essi guardano ad altre tipologie, che non mancano evidentemente dal gran corso della visualizzazione boccacciana (fin dalla precocissima testimonianza del ms. Paris, Bibliothèque Nationale, It. 482) [fig. 8]. 
Mi si permetta, in coda, di riportare alcune didascalie:

Corren una cortina y vese Crisanto, sentado en una silla, con un bufete delante y en él algunos libros, leyendo en uno.

Tocan chirimias, y córrese una cortina: aparece el rey Enrique durmiendo; delante, una mesa, con recado de escribir, y, a un lado, Ana Bolena.

Salen las Damas, córrese una cortina y estarón sentados el Rey y la Reina, con coronas y cetros, y la Infanta sentada, junto à la reina, y Volseo, detràs del Rey, en pie.

Tocan chirimias y clarines y salen a la jura los que pudieren, y el Rey y la Infan$t a$, que suben en un trono, ha da estar el cuerpo de Ana Bolena, cubierto con un tafetán, $Y$ en estando sentedo, la descubre.

...on tire la tapisserie er Mithridate paraît avec Hypsicraté et ses deux filles.

Pharnace entre dans la chambre où, la tapisserie tirée, il voit sur des trone set sa femme et ses soeurs à leurs pieds.

Queste descrizioni di aperture d'interni provengono da opere di drammaturghi del XVII secolo, per la precisione da Los dos amantes del cielo e La cisma de Angletera di Calderón e La mort de Mitrhidate di de la Calprènede. Li estraggo a mero titolo di esempio da un mazzo foltissimo di occorrenze e accosto un autore spagnolo a un autore francese per mostrare - ciò che il lettore avrà la bontà di accogliere sulla parola e che sarà da parte mia oggetto di dimostrazione in un lavoro acconcio- l'identità dell' uso in queste due culture. Identità che sposta o comunque indubbiamente arrichisce la complessità della questione. Per quello che è l'oggetto della dimostrazione da compiere altrove, mi sembra che la dipendenza della scena francese à compartiments si dia non rispetto allo spazio "medievale» a luoghi deputati, inventato come precedente della drammaturgia barocca dagli storici di fine Ottocento (per esempio da Eugène Rigal, fulminato dalla ricostruzione del palcoscenico di Valenciennes all'Exposition Universelle del 1878), ma dalla voga del modello spagnolo e dalle possibilità consentite dall'apertura delle cortine nello spazio del vestuario, prima che la cosiddetta âge classique combattasse la sua strenua battaglia contro questa cultura. $\mathrm{O}$ meglio, e più ampiamente: l'intreccio di questo modello scenico a quello della scenografia decorativa ed "apparente» di marca italiana mette in campo un'ipotesi storicamente più plausibile ed economica della tradizionale derivazione da un impianto scenico medievale, posto anche che le testimonianze francesi si dimostrano parallele al senso del passo di Leone de' Sommi a cui abbiamo già fatto riferimento, e che è venuto il momento di citare.

Chiede il primo al secondo interlocutore: 
Ora non ci volete voi dir cosa alcuna sopra quel modo di scene sfacciate o aperte, che dicono essersi alcune volte usate, e massimamente in Ispagna?

Conta riportare per intero l'illustrazione offerta nella risposta, dopo un necessario chiarimento relativo al significato dell'espressione (evidentemente non così chiaro e riferito a un' usanza non certo diffusa):

VERIDICO: Per scene aperte non intendente voi di quelle che si vede anco dentro alle lor stanze? e che di dentro a quelle alcuna volta si recita?

SANTINO: Di coteste chiedo io; che ve ne pare?

VERIDICO: Benché paia di certa vaghezza il vedersi in scena una camera aperta, ben parata, dentro alla quale (dirò così per esempio) uno amante si consulti con una ruffiana, e che paia aver del verisimile, è però tanto fuor del naturale essere la stanza senza il muro dinanzi (il che necessariamente far bisogna), che a me pare non molto convenirsi, oltra che, non so se il recitare in quel loco si potrà dire che sia in scena. Ben si potrìa, per fuggire questi due inconvenienti, aprir come una loggia od un verone, dove ricoresse alcuno a ragionare; ma non mi torrei però licenza di far stare nel resto voto il proscenio, e però condurci che i lor ragionamenti in tai lochi fossero sempre $\mathrm{o}$ in fine $\mathrm{o}$ in principio degl'atti; acciò che quasi di intermedio se li potesse dar nome. ${ }^{13}$

La pratica delle scene sfacciate è, dunque, qui dichiarata come recente ed estranea alle abitudini dello spettacolo italiano. Sembra, inoltre, che se ne offra una descrizione più per sentito dire che per pratica diretta («che dicono essersi alcune volte usate»), riferendola, come abbiamo detto, soprattutto alla Spagna ("massimamente in Ispagna»). Si tratta di una precisazione chiarissima e che, come tale, dovrebbe dissuadere da un uso indebito di questa testimonianza, con la riconduzione a una pratica «medievale».

Ma a questo mi dedicherò, come ho detto, altrove e un'altra volta, riflettendo soprattutto su quel prezioso documento rappresentato dal cosiddetto Mémoire de Mahelot, relativo agli apparati scenici dell'Hôtel de Bourgogne tra gli anni' 30 e '70 del XVII secolo. ${ }^{14}$

\section{VIII}

Allego, per ultima, una bislacca — quanto forse involontariamente profondavisualizzazione decameroniana. Il miniatore di questo Decameron - ancora francese del secondo quarto del quindicesimo secolo, conservato a Vienna— ha rinunciato a rappresentare qualsiasi pendenza narrativa della novella nona dell'ottava giornata, quella in cui lo stolido maestro Simone medico è beffato da Bruno e Buffalmacco e, credendo di andare a un'orgia satanica, è buttato dai buontem-

13. Ferruccio Marotti, Lo spettacolo dall'Umanesimo al Manierismo. Teoria e tecnica, Milano: Feltrinelli, 1974, p. 225-226.

14. Cfr. ora Le mémoire de Mahelot, édition critique établie et commentée par Pierre PASQUIER, Paris: Champion, 2005. 


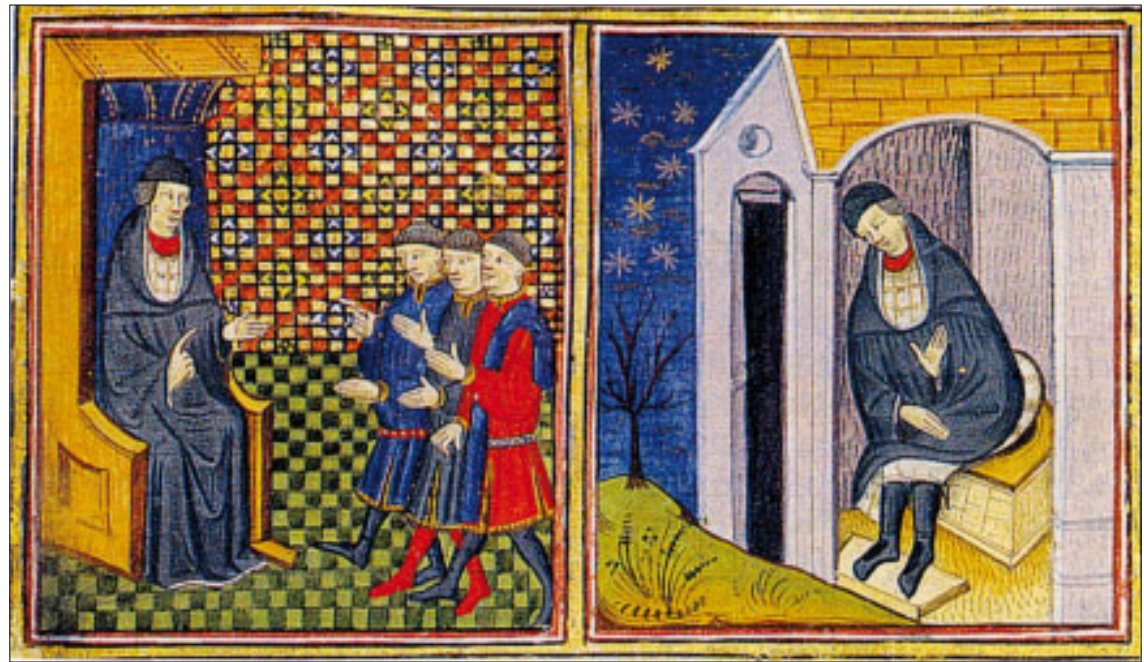

Fig. 9

poni in una fossa di bruttura, come era accaduto ad Andreuccio [fig. 9]. ${ }^{15}$ Qui il miniatore ha semplicemente contrapposto, in doppia vignetta autonoma, un maestro Simone in cattedra e in esercizio, e un maestro Simone a casa — con la parete debitamente squarciata - seduto al cacatoio, in un'occupazione dimessamente quotidiana. Una restituzione molto libera o senz'altro strampalata, che certo procede da distorsione e incomprensione del testo; ma tuttavia un'immagine esemplare per i tragitti del nostro entrare e uscire, del dentro e del fuori, e dell'idea che abbiamo provato a sostenere a proposito di un osceno - nel suo senso letterale di «fuori scena»— che comprende più ampiamente la dimensione della domesticità, fino alle uman privadi, per dirla con Dante. Qui forse sta il nesso più profondo della artificiosa restituzione che il teatro e la pittura si sono dati, in modi diversi e non confondibili, del mandato di figurare una historia. 